\title{
Measurement of the $\boldsymbol{\Omega}_{c}^{0}$ Baryon Lifetime
}

\author{
R. Aaij et al. \\ (LHCb Collaboration)
}

(Received 5 July 2018; revised manuscript received 31 July 2018; published 31 August 2018)

\begin{abstract}
We report a measurement of the lifetime of the $\Omega_{c}^{0}$ baryon using proton-proton collision data at center-ofmass energies of 7 and $8 \mathrm{TeV}$, corresponding to an integrated luminosity of $3.0 \mathrm{fb}^{-1}$ collected by the LHCb experiment. The sample consists of about $1000 \Omega_{b}^{-} \rightarrow \Omega_{c}^{0} \mu^{-} \bar{\nu}_{\mu} X$ signal decays, where the $\Omega_{c}^{0}$ baryon is detected in the $p K^{-} K^{-} \pi^{+}$final state and $X$ represents possible additional undetected particles in the decay. The $\Omega_{c}^{0}$ lifetime is measured to be $\tau_{\Omega_{c}^{0}}=268 \pm 24 \pm 10 \pm 2 \mathrm{fs}$, where the uncertainties are statistical, systematic, and from the uncertainty in the $D^{+}$lifetime, respectively. This value is nearly four times larger than, and inconsistent with, the current world-average value.
\end{abstract}

DOI: 10.1103/PhysRevLett.121.092003

Measurements of the lifetimes of hadrons containing heavy ( $b$ or $c$ ) quarks play an important role in testing theoretical approaches that are used to predict standard model parameters. The validation of such tools is important, as they can then be used to search for deviations from standard model expectations in other processes. One of the most predictive tools in quark flavor physics is the heavy quark expansion (HQE) [1-8], which describes the decay widths of hadrons containing heavy quarks, $Q$, through an expansion in powers of $1 / m_{Q}$, where $m_{Q}$ is the heavy quark mass. While predictions for absolute lifetimes carry relatively large uncertainties, ratios of lifetimes have smaller theoretical uncertainties [9]. Higher-order terms in the HQE are related to nonperturbative corrections, and to effects due to the presence of the other light quark(s) (spectator) in the heavy hadron. For beauty hadrons with a single heavy quark, these corrections are typically at the few percent level or less, due to the large mass of the $b$ quark [9]. For charm hadrons, since $m_{c}$ is significantly smaller than $m_{b}$, these higher-order corrections can be sizable. Therefore measurements of charm-hadron lifetimes provide a sensitive probe of their contributions [10-14].

While charm-meson lifetimes have been measured precisely and provide useful information on these higher-order terms, the knowledge of charm-baryon lifetimes is much less accurate. The lifetimes of the $D^{0}, D^{+}$, and $D_{s}^{+}$mesons are known to about $1 \%$ precision, whereas the corresponding uncertainties for the $\Lambda_{c}^{+}, \Xi_{c}^{+}, \Xi_{c}^{0}$, and $\Omega_{c}^{0}$ baryons are $3 \%, 6 \%, 10 \%$, and $17 \%$, respectively [15]. Improved

*Full author list given at the end of the article.

Published by the American Physical Society under the terms of the Creative Commons Attribution 4.0 International license. Further distribution of this work must maintain attribution to the author(s) and the published article's title, journal citation, and DOI. Funded by SCOAP ${ }^{3}$. measurements of the charm-baryon lifetimes provide complementary information to what can be gleaned from charm mesons. For example, contributions from $W$-exchange and constructive Pauli interference effects are present in charmbaryon decays, but are small or absent in charm-meson decays [11]. Moreover, for charm baryons, the spectator system may have spin $0\left(\Lambda_{c}^{+}, \Xi_{c}^{+}, \Xi_{c}^{0}\right)$ or spin $1\left(\Omega_{c}^{0}\right)$, whereas for charm mesons, the light quark spin is always equal to $1 / 2$.

It has been argued that the expected lifetime hierarchy, due to the higher order contributions discussed above, should be $[10-12,16-18]$

$$
\tau_{\Xi_{c}^{+}}>\tau_{\Lambda_{c}^{+}}>\tau_{\Xi_{c}^{0}}>\tau_{\Omega_{c}^{0}}
$$

The quark content of the $\Omega_{c}^{0}$ baryon is css, and the qualitative argument that the $\Omega_{c}^{0}$ lifetime should be the shortest is predicated on large constructive interference between the $s$ quark in the $c \rightarrow s W^{+}$transition in the $\Omega_{c}^{0}$ decay and the spectator $s$ quarks in the final state. However, it is also conceivable that the $\Omega_{c}^{0}$ lifetime could be the largest, depending on the treatment of higher-order terms in the HQE expansion [12].

Current measurements [15] are consistent with this hierarchy. The least well measured lifetime is that of the $\Omega_{c}^{0}$ baryon, with a value of $\tau_{\Omega_{c}^{0}}=69 \pm 12 \mathrm{fs}$, obtained by fixed-target experiments using a small number of signal decays [19-21].

In this Letter we report a new measurement of the $\Omega_{c}^{0}$ baryon lifetime using a sample of semileptonic (SL) $\Omega_{b}^{-} \rightarrow$ $\Omega_{c}^{0} \mu^{-} \bar{\nu}_{\mu} X$ decays, where the $\Omega_{c}^{0}$ baryons are detected in the $p K^{-} K^{-} \pi^{+}$final state and $X$ represents any additional undetected particles. Semileptonic $b$-meson decays were used previously by $\mathrm{LHCb}$ to make precise measurements of the $D_{s}^{+}$and $B_{s}^{0}$ lifetimes [22]. Throughout the text, chargeconjugate processes are implicitly included. 
To reduce the uncertainties associated with systematic effects, the lifetime ratio

$$
r_{\Omega_{c}^{0}} \equiv \frac{\tau_{\Omega_{c}^{0}}}{\tau_{D^{+}}}
$$

is measured, where the $D^{+}$meson is detected in $B \rightarrow$ $D^{+} \mu^{-} \bar{\nu}_{\mu} X$ decays, with $D^{+} \rightarrow K^{-} \pi^{+} \pi^{+}$. In the following, the symbols $H_{b}$ and $H_{c}$ are used to refer to the $b$ or $c$ hadron in either of the two modes indicated above.

The measurement uses proton-proton $(p p)$ collision data samples, collected by the LHCb experiment, corresponding to an integrated luminosity of $3.0 \mathrm{fb}^{-1}$, of which $1.0 \mathrm{fb}^{-1}$ was recorded at a center-of-mass energy of $7 \mathrm{TeV}$ and $2.0 \mathrm{fb}^{-1}$ at $8 \mathrm{TeV}$. The LHCb detector [23,24] is a singlearm forward spectrometer covering the pseudorapidity range $2<\eta<5$, designed for the study of particles containing $b$ or $c$ quarks. The tracking system provides a measurement of momentum $p$ of charged particles with a relative uncertainty that varies from $0.5 \%$ at low momentum to $1.0 \%$ at $200 \mathrm{GeV} / c$. The minimum distance of a track to a primary vertex (PV), the impact parameter (IP), is measured with a resolution of $\left(15+29 / p_{\mathrm{T}}\right) \mu \mathrm{m}$, where $p_{\mathrm{T}}$ is the component of the momentum transverse to the beam, in $\mathrm{GeV} / c$. Charged hadrons are identified using information from two ring-imaging Cherenkov ( $\mathrm{RICH}$ ) detectors [25]. Muons are identified by a system composed of alternating layers of iron and multiwire proportional chambers [26]. The online event selection is performed by a trigger [27], which consists of a hardware stage, based on information from the calorimeter and muon systems, followed by a software stage, which applies a full event reconstruction.

Simulation is required to model the effects of the detector acceptance and the imposed selection requirements. Proton-proton collisions are simulated using PYTHIA [28] with a specific LHCb configuration [29]. Decays of hadronic particles are described by EvTGEN [30], in which final-state radiation is generated using Рнотоs [31]. The interaction of the generated particles with the detector and its response are implemented using the GEANT4 toolkit [32] as described in Ref. [33].

Signal $\Omega_{b}^{-}$candidates are formed by combining an $\Omega_{c}^{0} \rightarrow p K^{-} K^{-} \pi^{+}$candidate with a $\mu^{-}$candidate. Each final-state particle in the decay is required to be detached from all PVs in the event, and is associated to the one with the smallest $\chi_{\mathrm{IP}}^{2}$. Here, $\chi_{\mathrm{IP}}^{2}$ is defined as the difference in $\chi^{2}$ of the particle's associated PV reconstructed with and without the considered track. The muon is required to have $p_{\mathrm{T}}>1 \mathrm{GeV} / c, p>6 \mathrm{GeV} / c$ and have particle identification (PID) information consistent with being a muon. The $\Omega_{c}^{0}$ candidate's decay products must have PID information consistent with their assumed particle hypotheses, and have $p_{\mathrm{T}}>0.25 \mathrm{GeV} / c$ and $p>2 \mathrm{GeV} / c$, except for the proton, which is required to have $p>8 \mathrm{GeV} / c$. To remove the contribution from promptly produced $\Omega_{c}^{0}$ baryons, each $\Omega_{c}^{0}$ candidate's reconstructed trajectory must not point back to any PV in the event. Only $\Omega_{c}^{0}$ candidates that have an invariant mass within $60 \mathrm{MeV} / c^{2}$ of the known $\Omega_{c}^{0}$ mass are retained.

The $\Omega_{c}^{0} \mu^{-}$combinations are required to form a good quality vertex and satisfy the invariant mass requirement, $m\left(\Omega_{c}^{0} \mu^{-}\right)<8.0 \mathrm{GeV} / c^{2}$. Random combinations of $\Omega_{c}^{0}$ and $\mu^{-}$are suppressed by requiring the fitted $z$ coordinates of the $\Omega_{c}^{0}$ and $\Omega_{b}^{-}$decay vertices to satisfy $z\left(\Omega_{c}^{0}\right)-z\left(\Omega_{c}^{0} \mu^{-}\right)>$ $-0.05 \mathrm{~mm}$, where the $z$ axis is parallel to the beam direction.

To ensure precise modeling of the decay-time acceptance from simulation, the candidates must satisfy a well-defined set of hardware and software trigger requirements. At the hardware level, candidates are required to pass the singlemuon trigger, and, at the software level, to pass specific triggers designed to select multi-body final states containing a muon [27].

To improve the signal-to-background ratio in the $\Omega_{c}^{0} \mu^{-}$ sample, a boosted decision tree (BDT) discriminant [34,35] is built from 18 variables, which include the $\chi^{2}$ for the $\Omega_{b}^{-}$ and $\Omega_{c}^{0}$ decay-vertex fits, and $\chi_{\mathrm{IP}}^{2}, p, p_{\mathrm{T}}$, and a PID response variable for each final-state hadron. The BDT is trained using simulated $\Omega_{b}^{-} \rightarrow \Omega_{c}^{0} \mu^{-} \bar{\nu}_{\mu} X$ decays for the signal, while background is taken from the $\Omega_{c}^{0}$ mass sidebands, $30<\left|m\left(p K^{-} K^{-} \pi^{+}\right)-m_{\Omega_{c}^{0}}\right|<50 \mathrm{MeV} / c^{2}$, where $m_{\Omega_{c}^{0}}$ is the known $\Omega_{c}^{0}$ mass [15]. The requirement on the BDT response is determined by optimizing the figure of merit $S / \sqrt{S+B}$, where $S$ and $B$ are the expected signal and background yields within a $\pm 15 \mathrm{MeV} / c^{2}$ mass region centered on the mass peak, respectively. The optimal BDT requirement provides a signal (background) efficiency of $78 \%(16 \%)$.

The $D^{+} \mu^{-}$candidates, used for normalization, are formed by combining $D^{+} \rightarrow K^{-} \pi^{+} \pi^{+}$and $\mu^{-}$candidates. The selections are identical to those discussed above, except the mass window is centered on the known $D^{+}$mass and the BDT requirement is eliminated. Only $10 \%$ of the $D^{+} \mu^{-}$data, selected at random, are used in the analysis, since the full sample is much larger than needed for this measurement.

The invariant-mass distributions for the selected $\Omega_{c}^{0}$ and $D^{+}$candidates in the two $H_{c} \mu^{-}$final states are shown in Fig. 1. Both distributions are fitted using the sum of a signal component, defined as the sum of two Gaussian functions with a common mean, and an exponential shape to represent the combinatorial background. From a binned maximum-likelihood fit, the fitted $\Omega_{c}^{0} \mu^{-}$and $D^{+} \mu^{-}$yields are $978 \pm 60$ and $(809 \pm 1) \times 10^{3}$, respectively. The number of $\Omega_{c}^{0}$ signal decays is at least an order of magnitude larger than any previous sample used for an $\Omega_{c}^{0}$ lifetime measurement.

The decay time of each $H_{c}$ candidate is determined from the positions of the $H_{b}$ and $H_{c}$ decay vertices, and 

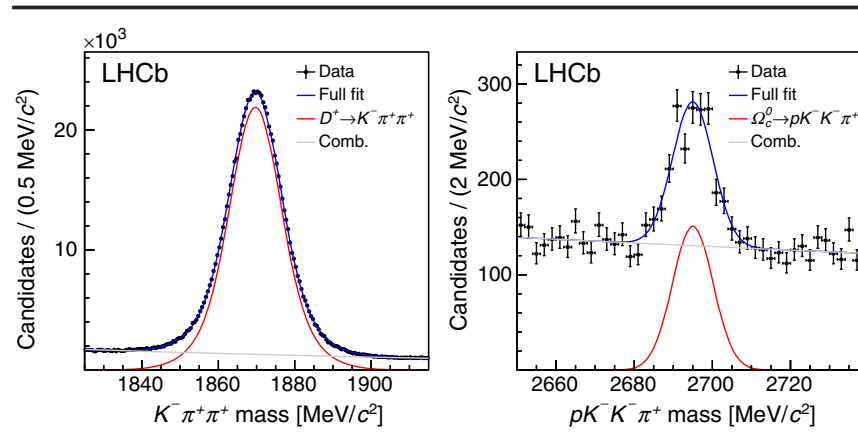

FIG. 1. Invariant-mass distributions for (left) $D^{+}$candidates in $B \rightarrow D^{+} \mu^{-} \bar{\nu}_{\mu} X$ decays and (right) $\Omega_{c}^{0}$ candidates in $\Omega_{b}^{-} \rightarrow$ $\Omega_{c}^{0} \mu^{-} \bar{\nu}_{\mu} X$ decays. The results of the fits, as described in the text, are overlaid.

the measured $H_{c}$ momentum. The background-subtracted decay-time spectra are obtained using the sPlot technique [36], where the measured $H_{c}$ mass is used as the discriminating variable. The uncertainties in the bin-by-bin signal yields reflect both the finite signal yield and the statistical uncertainty due to the background subtraction.

Potential backgrounds from (i) random $H_{c} \mu^{-}$combinations, (ii) $H_{b} \rightarrow H_{c} \tau^{-} \bar{\nu}_{\tau}, \quad \tau^{-} \rightarrow \mu^{-} \nu_{\tau} \bar{\nu}_{\mu}$ decays, and (iii) $H_{b} \rightarrow H_{c} \bar{D}, \bar{D} \rightarrow \mu^{-} X$, where $\bar{D}$ represents a $D_{s}^{-}$, $D^{-}$or $\bar{D}^{0}$ meson, could lead to a bias on the lifetime, since the muon is not produced directly at the $H_{b}$ decay vertex. These backgrounds have been investigated and constitute a small fraction of the observed signal, about $3 \%$ in total, and have decay-time spectra that are similar to the true $H_{c} \mu^{-} \bar{\nu}_{\mu}$ final state due to the $\chi^{2}$ requirements on the $H_{b}$ vertex fit. Moreover, these backgrounds affect the signal and the normalization mode similarly, thus leading to at least a partial cancellation of any bias. Contamination in the $\Omega_{b}^{-} \rightarrow \Omega_{c}^{0} \mu^{-} \bar{\nu}_{\mu} X$ sample from misidentified four-body $D^{0}$ final states in $B \rightarrow D^{0} \mu^{-} \bar{\nu}_{\mu} X$ decays has been investigated, and none are found to peak in the $\Omega_{c}^{0}$ signal region.

The decay-time spectra for the $\Omega_{c}^{0}$ and $D^{+}$signals are shown in Fig. 2, along with the results of the fits described below. The decrease in the signal yield as the decay time approaches zero is mainly due to the effects of the $H_{c}$ decay-time resolution, which is in the range of 85-100 fs, and the $z\left(H_{c}\right)-z\left(H_{c} \mu^{-}\right)>-0.05 \mathrm{~mm}$ requirement.

The decay-time signal model $S\left(t_{\text {rec }}\right)$ takes the form

$$
S\left(t_{\text {rec }}\right)=f\left(t_{\text {rec }}\right) g\left(t_{\text {rec }}\right) \beta\left(t_{\text {rec }}\right) .
$$

Here, $f\left(t_{\text {rec }}\right)$ is a signal template of reconstructed decay times, obtained from the full LHCb simulation, after all selections have been applied as in the data. The signal template is multiplied by $g\left(t_{\text {rec }}\right)=\exp \left(-t_{\text {rec }} / \tau_{\text {fit }}^{H_{c}}\right) /$ $\exp \left(-t_{\text {rec }} / \tau_{\text {sim }}^{H_{c}}\right)$, where $\tau_{\text {sim }}^{D^{+}}=1040$ fs and $\tau_{\text {sim }}^{\Omega_{c}^{0}}=250 \mathrm{fs}$ are the lifetimes used in the simulation, and $\tau_{\text {fit }}^{H_{c}}$ is the signal lifetime to be fitted. The function $\beta\left(t_{\text {rec }}\right)$ is a correction that
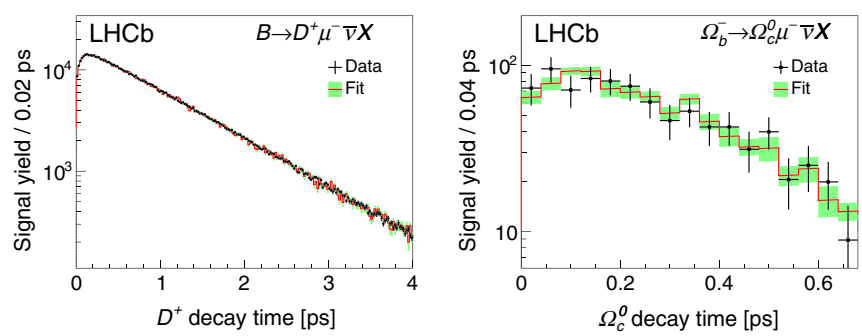

FIG. 2. Decay-time spectra for (left) $D^{+}$signal in $B \rightarrow D^{+} \mu^{-} X$ events and (right) $\Omega_{c}^{0}$ signal in $\Omega_{b}^{-} \rightarrow \Omega_{c}^{0} \mu^{-} X$ events. Overlaid are the fit results, as described in the text, along with the uncertainties due to finite simulated sample sizes.

accounts for a small difference in the efficiency between data and simulation for reconstructing tracks in the vertex detector that originate far from the beam line [37].

Given the precise knowledge of the $D^{+}$meson lifetime (1040 $\pm 7 \mathrm{fs}$ ) [15], the $D^{+} \mu^{-}$sample is used to calibrate $\beta\left(t_{\mathrm{rec}}\right)$ and validate the fit. The signal template is obtained from simulated $B \rightarrow D^{+} \mu^{-} \bar{\nu}_{\mu} X$ decays, where contributions from $B \rightarrow D^{+} \tau^{-} \bar{\nu}_{\tau} X$ decays are included. The function $\beta\left(t_{\text {rec }}\right)$ is obtained by taking the ratio between the $D^{+}$ decay-time spectrum in data (obtained via the sPlot technique) and that obtained from simulation. The ratio shows a linear dependence, and a fit to the function $\beta\left(t_{\mathrm{rec}}\right)=1+\beta_{0} t_{\mathrm{rec}}$ yields $\beta_{0}=(-0.89 \pm 0.32) \times 10^{-2} \mathrm{ps}^{-1}$. If the $\beta\left(t_{\text {rec }}\right)$ function is excluded from the fit, $\tau_{\text {fit }}^{D^{+}}$is $10 \mathrm{fs}$ below the world average. The result of the binned $\chi^{2}$ fit after this correction is applied is shown in Fig. 2 (left), where the fitted lifetime is found to be $\tau_{\text {fit }}^{D^{+}}=1042.0 \pm 1.7$ (stat) fs.

The $\Omega_{c}^{0}$ lifetime is determined from a simultaneous fit to the $\Omega_{c}^{0}$ and $D^{+}$decay-time spectra, for which the free parameters in the fit are $r_{\Omega_{c}^{0}}$ [see Eq. (2)] and $\tau_{\text {fit }}^{D^{+}}$. By fitting for the ratio $r_{\Omega_{c}^{0}}$, correlated systematic uncertainties partially cancel. In the $\Omega_{c}^{0}$ decay-time fit, $\beta_{0}$ is scaled by $4 / 3$ since the effect is expected to scale with the number of charged final state particles in the $H_{c}$ decay [37]. The simulation includes contributions from $\Omega_{c}^{0} \tau^{-} \bar{\nu}_{\tau} X$ final states. The results of the fit to the $\Omega_{c}^{0}$ decay-time distribution are shown in Fig. 2 (right), where the value $r_{\Omega_{c}^{0}}=0.258 \pm 0.023$ (stat) is obtained. Multiplying this value by $\tau^{D^{+}}=1040 \mathrm{fs}$ [15], the $\Omega_{c}^{0}$ lifetime is measured to be $268 \pm 24 \mathrm{fs}$. This is about four times larger than, and incompatible with, the current world average value of $69 \pm 12$ fs [15].

Several cross-checks have been performed to ensure the robustness of this result. To confirm that the signal events are from SL $\Omega_{b}^{-}$decays, a number of distributions, such as the $\Omega_{c}^{0} \mu^{-}$mass spectrum, $p_{\mathrm{T}}$ and decay time have been compared between data (using sPlot) and the $\Omega_{b}^{-} \rightarrow$ $\Omega_{c}^{0} \mu^{-} \bar{\nu}_{\mu} X$ simulation. In all cases, good agreement is found. The lifetime measurement has also been performed using a 
simple subtraction of the $\Omega_{c}^{0}$ mass sidebands, and we find good agreement with the value obtained by the sPlot technique. The $\Omega_{c}^{0}$ decay-time distribution obtained from an independent and comparably sized data sample of semileptonic decays collected at $13 \mathrm{TeV}$ center-of-mass energy has been examined, and the distribution is consistent with the one observed here. The procedure has also been checked using a sample of about $88000 B^{-} \rightarrow D^{0}(\rightarrow$ $\left.K^{+} K^{-} \pi^{+} \pi^{-}\right) \mu^{-} X$ decays to measure the $D^{0}$ meson lifetime. The obtained lifetime is consistent with the expected value within about one standard deviation. The analysis has also been carried out with either tighter PID or tighter BDT requirements, and the fitted $\Omega_{c}^{0}$ lifetime in each case is consistent with the value from the default fit. The analysis has also been checked with the $\Lambda_{c}^{+}$baryon, and the lifetime is consistent with expectations.

A number of sources of systematic uncertainty on the measured ratio $r_{\Omega_{c}^{0}}$ have been investigated, and are summarized in Table I. The decay time acceptance correction $\beta\left(t_{\text {rec }}\right)$ leads to an uncertainty of $0.5 \%$ on $r_{\Omega_{c}^{0}}$, which includes a contribution from the finite sample sizes and the choice of fit function.

Studies of the $D^{+}$calibration mode show a small dependence of the $\beta_{0}$ parameter on the $p_{\mathrm{T}}$ and $\eta$ of the $H_{b}$ hadron. In the case that the $p_{\mathrm{T}}$ and $\eta$ spectra in data and simulation differ, it could cause a shift in the average $\beta_{0}$. The uncertainty on $r_{\Omega_{c}^{0}}$ is obtained by taking into account the variation of $\beta_{0}$ in different $p_{\mathrm{T}}$ and $\eta$ ranges, and the extent to which the $p_{\mathrm{T}}$ and $\eta$ spectra may differ between data and simulation.

The world-average value of the $\Omega_{b}^{-}$lifetime is $1.64_{-0.17}^{+0.18} \mathrm{ps}$ [15], whereas the simulation uses $1.60 \mathrm{ps}$. To assess the potential impact on the $\Omega_{c}^{0}$ lifetime, we weight $f\left(t_{\text {rec }}\right)$ to replicate an $\Omega_{b}^{-}$lifetime of either 1.50 or $1.70 \mathrm{ps}$. The changes in $r_{\Omega_{c}^{0}}$ are assigned as a systematic uncertainty.

The decay-time resolution has been checked by comparing the $D^{0}$ decay-time spectra in $B^{-} \rightarrow D^{0} \pi^{-}$decays, where no explicit requirement on the flight distance of the $D^{0}$ is applied. Negative decay times are entirely due to

TABLE I. Summary of systematic uncertainties on the lifetime ratio, $r_{\Omega_{c}^{0}}$, in units of $10^{-4}$.

\begin{tabular}{lc}
\hline \hline Source & $r_{\Omega_{c}^{0}}\left(10^{-4}\right)$ \\
\hline Decay-time acceptance & 13 \\
$\Omega_{b}^{-}$production spectrum & 3 \\
$\Omega_{b}^{-}$lifetime & 4 \\
Decay-time resolution & 3 \\
Background subtraction & 18 \\
$H_{c}\left(\tau^{-}, D\right)$, random $\mu^{-}$ & 8 \\
Simulated sample size & 98 \\
Total systematic & 101 \\
Statistical uncertainty & 230 \\
\hline \hline
\end{tabular}

the decay-time resolution, and simulation is found to agree well with data. To assess a potential impact of a small difference in decay-time resolution between simulation and data, new $\Omega_{c}^{0}$ and $D^{+}$signal templates are formed where the reconstructed decay time is smeared by an additional $15 \%$, beyond what is produced by the full simulation. The fit is redone, and the difference in $r_{\Omega_{c}^{0}}$ from the nominal value is assigned as a systematic uncertainty.

The method for background subtraction uses the sPlot technique, which has some dependence on the choice of signal and background functions. To assess a potential systematic effect, the decay-time spectra are obtained using a sideband subtraction of the $H_{c}$ mass spectra for both the signal and the normalization modes. The sideband-subtracted decay-time spectra are then fitted using the decaytime fit described above. The difference between this result and the nominal one is assigned as a systematic uncertainty.

The decay-time spectra in both $\Omega_{c}^{0} \mu^{-}$and $D^{+} \mu^{-}$samples have small contributions from random combinations of $H_{c}$ and $\mu^{-}$candidates $[(0.8 \pm 0.2) \%$ of the signal], as well as physics backgrounds where the $\mu^{-}$comes from either a $\tau^{-}$ $[(1.8 \pm 0.3 \%)]$ or a SL $D$ decay $[(0.5 \pm 0.2) \%]$. From simulation and data control samples, we find that the effective lifetimes of these backgrounds are within $10 \%$ of the true signal lifetime; this is due to the requirement that the muon candidate must form a good vertex with the $H_{c}$ candidate. The impact on the $\Omega_{c}^{0}$ lifetime is evaluated using pseudoexperiments, where mixtures of these backgrounds (with different decay-time spectra) and signal decays are formed and fitted assuming a single lifetime for the sample. The difference in the mean value of $r_{\Omega_{c}^{0}}$ between the nominal fit, and that with the backgrounds added is assigned as the systematic uncertainty.

The systematic uncertainty due to the finite size of the simulated samples is assessed by repeating the fit to the data many times, where in each fit the simulated-template bin contents are fluctuated within their uncertainties. The standard deviation of the distribution of the fitted $r_{\Omega_{c}^{0}}$ values is assigned as a systematic uncertainty.

In summary, we use $p p$ collision data samples at 7 and $8 \mathrm{TeV}$ center-of-mass energies, corresponding to $3.0 \mathrm{fb}^{-1}$ of integrated luminosity, to measure the lifetime of the $\Omega_{c}^{0}$ baryon. The measured ratio of lifetimes and absolute $\Omega_{c}^{0}$ lifetime are

$$
\begin{aligned}
& \frac{\tau_{\Omega_{c}^{0}}}{\tau_{D^{+}}}=0.258 \pm 0.023 \pm 0.010, \\
& \tau_{\Omega_{c}^{0}}=268 \pm 24 \pm 10 \pm 2 \mathrm{fs},
\end{aligned}
$$

where the first uncertainty is statistical, the second is systematic, and the third is due to the uncertainty in the $D^{+}$lifetime [15]. The measured $\Omega_{c}^{0}$ lifetime is about four times larger than, and inconsistent with, the world average value of $69 \pm 12 \mathrm{fs}$ [15]. 
With this measurement, the lifetime hierarchy places the $\Omega_{c}^{0}$ baryon as having the second largest lifetime after the $\Xi_{c}^{+}$baryon,

$$
\tau_{\Xi_{c}^{+}}>\tau_{\Omega_{c}^{0}}>\tau_{\Lambda_{c}^{+}}>\tau_{\Xi_{c}^{0}}
$$

The result presented here may suggest that the constructive interference between the $s$ quark in the $c \rightarrow s W^{+}$transition in the $\Omega_{c}^{0}$ decay and the spectator $s$ quarks in the final state is smaller than expected, that the spin of the ss system plays a larger role, or that additional or higher order contributions in the heavy quark expansion need to be considered.

We express our gratitude to our colleagues in the CERN accelerator departments for the excellent performance of the LHC. We thank the technical and administrative staff at the LHCb institutes. We acknowledge support from CERN and from the national agencies: CAPES, CNPq, FAPERJ and FINEP (Brazil); MOST and NSFC (China); CNRS/ IN2P3 (France); BMBF, DFG and MPG (Germany); INFN (Italy); NWO (Netherlands); MNiSW and NCN (Poland); MEN/IFA (Romania); MinES and FASO (Russia); MinECo (Spain); SNSF and SER (Switzerland); NASU (Ukraine); STFC (United Kingdom); NSF (USA). We acknowledge the computing resources that are provided by CERN, IN2P3 (France), KIT and DESY (Germany), INFN (Italy), SURF (Netherlands), PIC (Spain), GridPP (United Kingdom), RRCKI and Yandex LLC (Russia), CSCS (Switzerland), IFIN-HH (Romania), CBPF (Brazil), PL-GRID (Poland) and OSC (USA). We are indebted to the communities behind the multiple open-source software packages on which we depend. Individual groups or members have received support from AvH Foundation (Germany); EPLANET, Marie Skłodowska-Curie Actions and ERC (European Union); ANR, Labex P2IO and OCEVU, and Région Auvergne-Rhóne-Alpes (France); Key Research Program of Frontier Sciences of CAS, CAS PIFI, and the Thousand Talents Program (China); RFBR, RSF and Yandex LLC (Russia); GVA, XuntaGal and GENCAT (Spain); Herchel Smith Fund, the Royal Society, the English-Speaking Union and the Leverhulme Trust (United Kingdom); Laboratory Directed Research and Development program of LANL (USA).

[1] V. A. Khoze and M. A. Shifman, Heavy quarks, Sov. Phys. Usp. 26, 387 (1983).

[2] I. I. Y. Bigi and N. G. Uraltsev, Gluonic enhancements in non-spectator beauty decays - an inclusive mirage though an exclusive possibility, Phys. Lett. B 280, 271 (1992).

[3] I. I. Bigi, N. G. Uraltsev, and A. I. Vainshtein, Nonperturbative corrections to inclusive beauty and charm decays: QCD versus phenomenological models, Phys. Lett. B 293, 430 (1992); Erratum, Phys. Lett. B 297, 477(E) (1992).

[4] B. Blok and M. Shifman, The rule of discarding $1 / N_{c}$ in inclusive weak decays (I), Nucl. Phys. B399, 441 (1993).
[5] B. Blok and M. Shifman, The rule of discarding $1 / N_{c}$ in inclusive weak decays (II), Nucl. Phys. B399, 459 (1993).

[6] M. Neubert, B decays and the heavy quark expansion, Adv. Ser. Dir. High Energy Phys. 15, 239 (1998).

[7] N. Uraltsev, Heavy quark expansion in beauty and its decays, arXiv:hep-ph/9804275; also published in Proceedings, Heavy Flavour Physics: A Probe of Nature's Grand Design, Proc. Intern. School of Physics "Enrico Fermi," Course CXXXVII, edited by I. Bigi and L. Moroni (IOS Press, Amsterdam, 1998).

[8] I. I. Bigi, The QCD perspective on lifetimes of heavy flavor hadrons, arXiv:hep-ph/9508408.

[9] A. Lenz, Lifetimes and heavy quark expansion, Int. J. Mod. Phys. A 30, 1543005 (2015).

[10] H.-Y. Cheng, Charmed baryons circa 2015, Front. Phys. (Beijing) 10, 101406 (2015).

[11] S. Bianco, F. L. Fabbri, D. Benson, and I. Bigi, A cicerone for the physics of charm, Riv. Nuovo Cimento 26N7, 1 (2003).

[12] B. Blok and M. A. Shifman, Lifetimes of charmed hadrons revisited. Facts and fancy, arXiv:hep-ph/9311331.

[13] M. Kirk, A. Lenz, and T. Rauh, Dimension-six matrix elements for meson mixing and lifetimes from sum rules, J. High Energy Phys. 12 (2017) 068.

[14] A. Lenz and T. Rauh, D-meson lifetimes within the heavy quark expansion, Phys. Rev. D 88, 034004 (2013).

[15] M. Tanabashi et al. (Particle Data Group), Review of particle physics, Phys. Rev. D98, 030001 (2018).

[16] H.-Y. Cheng, A phenomenological analysis of heavy hadron lifetimes, Phys. Rev. D 56, 2783 (1997).

[17] G. Bellini, I. I. Y. Bigi, and P. J. Dornan, Lifetimes of charm and beauty hadrons, Phys. Rep. 289, 1 (1997).

[18] M. A. Shifman and M. B. Voloshin, Preasymptotic effects in inclusive weak decays of charmed particles, Yad. Fiz. 41, 187 (1985) [Sov. J. Nucl. Phys. 41, 120 (1985)].

[19] J. M. Link et al. (FOCUS Collaboration), Measurement of the $\Omega_{c}^{0}$ lifetime, Phys. Lett. B 561, 41 (2003).

[20] M. I. Adamovich et al. (WA89 Collaboration), Measurement of the $\Omega_{c}^{0}$ lifetime, Phys. Lett. B 358, 151 (1995).

[21] P. L. Frabetti et al. (E687 Collaboration), First measurement of the lifetime of the $\Omega_{c}^{0}$, Phys. Lett. B 357, 678 (1995).

[22] R. Aaij et al. (LHCb Collaboration), Measurement of $B_{s}^{0}$ and $D_{s}^{-}$Meson Lifetimes, Phys. Rev. Lett. 119, 101801 (2017).

[23] A. A. Alves Jr. et al. (LHCb Collaboration), The LHCb detector at the LHC, J. Instrum. 3, S08005 (2008).

[24] R. Aaij et al. (LHCb Collaboration), LHCb detector performance, Int. J. Mod. Phys. A 30, 1530022 (2015).

[25] M. Adinolfi et al., Performance of the LHCb RICH detector at the LHC, Eur. Phys. J. C 73, 2431 (2013).

[26] F. Archilli et al., Performance of the muon identification at LHCb, J. Instrum. 8, P10020 (2013).

[27] R. Aaij et al., The LHCb trigger and its performance in 2011, J. Instrum. 8, P04022 (2013).

[28] T. Sjöstrand, S. Mrenna, and P. Skands, PYTHIA 6.4 physics and manual, J. High Energy Phys. 05 (2006) 026; A brief introduction to PYTHIA 8.1, Comput. Phys. Commun. 178, 852 (2008). 
[29] I. Belyaev et al., Handling of the generation of primary events in Gauss, the LHCb simulation framework, J. Phys. Conf. Ser. 331, 032047 (2011).

[30] D. J. Lange, The EvtGen particle decay simulation package, Nucl. Instrum. Methods Phys. Res., Sect. A 462, 152 (2001).

[31] P. Golonka and Z. Was, PHOTOS Monte Carlo: A precision tool for QED corrections in $Z$ and $W$ decays, Eur. Phys. J. C 45, 97 (2006).

[32] J. Allison et al. (Geant4 Collaboration), Geant4 developments and applications, IEEE Trans. Nucl. Sci. 53, 270 (2006); S. Agostinelli et al. (Geant4 Collaboration), Geant4: A simulation toolkit, Nucl. Instrum. Methods Phys. Res., Sect. A 506, 250 (2003).

[33] M. Clemencic, G. Corti, S. Easo, C. R. Jones, S. Miglioranzi, M. Pappagallo, and P. Robbe, The LHCb simulation application, Gauss: Design, evolution and experience, J. Phys. Conf. Ser. 331, 032023 (2011).

[34] L. Breiman, J. H. Friedman, R. A. Olshen, and C. J. Stone, Classification and Regression Trees (Wadsworth International Group, Belmont, 1984).

[35] Y. Freund and R. E. Schapire, A decision-theoretic generalization of on-line learning and an application to boosting, J. Comput. Syst. Sci. 55, 119 (1997).

[36] M. Pivk and F. R. Le Diberder, sPlot: A statistical tool to unfold data distributions, Nucl. Instrum. Methods A555, 356 (2005).

[37] R. Aaij et al. (LHCb Collaboration), Measurements of the $B^{+}, B^{0}, B_{s}^{0}$ meson and $\Lambda_{b}^{0}$ baryon lifetimes, J. High Energy Phys. 04 (2014) 114.

R. Aaij ${ }^{27}$ B. Adeva, ${ }^{41}$ M. Adinolfi, ${ }^{48}$ C. A. Aidala,${ }^{73}$ Z. Ajaltouni, ${ }^{5}$ S. Akar, ${ }^{59}$ P. Albicocco, ${ }^{18}$ J. Albrecht,${ }^{10}$ F. Alessio, ${ }^{42}$ M. Alexander, ${ }^{53}$ A. Alfonso Albero, ${ }^{40} \mathrm{~S}$. Ali, ${ }^{27} \mathrm{G}$. Alkhazov, ${ }^{33} \mathrm{P}$. Alvarez Cartelle, ${ }^{55}$ A. A. Alves Jr, ${ }^{41} \mathrm{~S}$. Amato, ${ }^{2}$ S. Amerio, ${ }^{23}$ Y. Amhis, ${ }^{7}$ L. An,${ }^{3}$ L. Anderlini, ${ }^{17}$ G. Andreassi, ${ }^{43}$ M. Andreotti, ${ }^{16, a}$ J. E. Andrews,${ }^{60}$ R. B. Appleby, ${ }^{56}$ F. Archilli, ${ }^{27}$ P. d'Argent, ${ }^{12}$ J. Arnau Romeu, ${ }^{6}$ A. Artamonov, ${ }^{39}$ M. Artuso, ${ }^{61}$ K. Arzymatov,${ }^{37}$ E. Aslanides,${ }^{6}$ M. Atzeni, ${ }^{44}$ B. Audurier, ${ }^{22}$ S. Bachmann, ${ }^{12}$ J. J. Back,${ }^{50}$ S. Baker, ${ }^{55}$ V. Balagura, ${ }^{7, b}$ W. Baldini, ${ }^{16}$ A. Baranov, ${ }^{37}$ R. J. Barlow, ${ }^{56}$ S. Barsuk, ${ }^{7}$ W. Barter, ${ }^{56}$ F. Baryshnikov, ${ }^{70}$ V. Batozskaya, ${ }^{31}$ B. Batsukh, ${ }^{61}$ V. Battista, ${ }^{43}$ A. Bay, ${ }^{43}$ J. Beddow, ${ }^{53}$ F. Bedeschi, ${ }^{24}$ I. Bediaga, ${ }^{1}$ A. Beiter, ${ }^{61}$ L. J. Bel, ${ }^{27}$ S. Belin, ${ }^{22}$ N. Beliy, ${ }^{63}$ V. Bellee ${ }^{43}$ N. Belloli, ${ }^{20, c}$ K. Belous, ${ }^{39}$ I. Belyaev, ${ }^{34,42}$ E. Ben-Haim, ${ }^{8}$ G. Bencivenni, ${ }^{18}$ S. Benson, ${ }^{27}$ S. Beranek, ${ }^{9}$ A. Berezhnoy, ${ }^{35}$ R. Bernet, ${ }^{44}$ D. Berninghoff, ${ }^{12}$ E. Bertholet, ${ }^{8}$ A. Bertolin, ${ }^{23}$ C. Betancourt, ${ }^{44}$ F. Betti,${ }^{15,42}$ M. O. Bettler, ${ }^{49}$ M. van Beuzekom, ${ }^{27}$ Ia. Bezshyiko, ${ }^{44}$ S. Bhasin,${ }^{48}$ J. Bhom, ${ }^{29}$ S. Bifani,${ }^{47}$ P. Billoir, ${ }^{8}$ A. Birnkraut, ${ }^{10}$ A. Bizzeti, ${ }^{17, d}$ M. Bjørn,${ }^{57}$ M. P. Blago, ${ }^{42}$ T. Blake, ${ }^{50}$ F. Blanc, ${ }^{43}$ S. Blusk, ${ }^{61}$ D. Bobulska, ${ }^{53}$ V. Bocci, ${ }^{26}$ O. Boente Garcia, ${ }^{41}$ T. Boettcher, ${ }^{58}$ A. Bondar,${ }^{38, e}$ N. Bondar, ${ }^{33}$ S. Borghi,${ }^{56,42}$ M. Borisyak ${ }^{37}$ M. Borsato, ${ }^{41}$ F. Bossu, ${ }^{7}$ M. Boubdir, ${ }^{9}$ T. J. V. Bowcock, ${ }^{54}$ C. Bozzi,${ }^{16,42}$ S. Braun, ${ }^{12}$ M. Brodski, ${ }^{42}$ J. Brodzicka, ${ }^{29}$ A. Brossa Gonzalo, ${ }^{50}$ D. Brundu, ${ }^{22}$ E. Buchanan, ${ }^{48}$ A. Buonaura, ${ }^{44}$ C. Burr, ${ }^{56}$ A. Bursche, ${ }^{22}$ J. Buytaert, ${ }^{42}$ W. Byczynski, ${ }^{42}$ S. Cadeddu, ${ }^{22}$ H. Cai, ${ }^{64}$ R. Calabrese, ${ }^{16, a}$ R. Calladine, ${ }^{47}$ M. Calvi, ${ }^{20, c}$ M. Calvo Gomez ${ }^{40, f}$ A. Camboni,${ }^{40, f}$ P. Campana ${ }^{18}$ D. H. Campora Perez, ${ }^{42}$ L. Capriotti, ${ }^{56}$ A. Carbone, ${ }^{15, g}$ G. Carboni, ${ }^{25}$ R. Cardinale, ${ }^{19, h}$ A. Cardini, ${ }^{22}$ P. Carniti, ${ }^{20, c}$

L. Carson, ${ }^{52}$ K. Carvalho Akiba, ${ }^{2}$ G. Casse,${ }^{54}$ L. Cassina, ${ }^{20}$ M. Cattaneo ${ }^{42}$ G. Cavallero, ${ }^{19, \mathrm{~h}}$ R. Cenci, ${ }^{24, \mathrm{i}}$ D. Chamont, ${ }^{7}$ M. G. Chapman, ${ }^{48}$ M. Charles, ${ }^{8}$ Ph. Charpentier, ${ }^{42}$ G. Chatzikonstantinidis, ${ }^{47}$ M. Chefdeville, ${ }^{4}$ V. Chekalina,${ }^{37}$ C. Chen, ${ }^{3}$ S. Chen, ${ }^{22}$ S.-G. Chitic, ${ }^{42}$ V. Chobanova, ${ }^{41}$ M. Chrzaszcz, ${ }^{42}$ A. Chubykin, ${ }^{33}$ P. Ciambrone, ${ }^{18}$ X. Cid Vidal, ${ }^{41}$ G. Ciezarek, ${ }^{42}$ P. E. L. Clarke, ${ }^{52}$ M. Clemencic, ${ }^{42}$ H. V. Cliff, ${ }^{49}$ J. Closier, ${ }^{42}$ V. Coco, ${ }^{42}$ J. A. B. Coelho, ${ }^{7}$ J. Cogan, ${ }^{6}$ E. Cogneras, ${ }^{5}$

L. Cojocariu, ${ }^{32}$ P. Collins, ${ }^{42}$ T. Colombo, ${ }^{42}$ A. Comerma-Montells, ${ }^{12}$ A. Contu, ${ }^{22}$ G. Coombs, ${ }^{42}$ S. Coquereau, ${ }^{40}$ G. Corti, ${ }^{42}$ M. Corvo, ${ }^{16, a}$ C. M. Costa Sobral, ${ }^{50}$ B. Couturier ${ }^{42}$ G. A. Cowan, ${ }^{52}$ D. C. Craik,${ }^{58}$ A. Crocombe, ${ }^{50}$ M. Cruz Torres, ${ }^{1}$ R. Currie, ${ }^{52}$ C. D’Ambrosio, ${ }^{42}$ F. Da Cunha Marinho, ${ }^{2}$ C. L. Da Silva, ${ }^{74}$ E. Dall'Occo, ${ }^{27}$ J. Dalseno, ${ }^{48}$ A. Danilina, ${ }^{34}$ A. Davis, ${ }^{3}$ O. De Aguiar Francisco, ${ }^{42}$ K. De Bruyn, ${ }^{42}$ S. De Capua, ${ }^{56}$ M. De Cian, ${ }^{43}$ J. M. De Miranda, ${ }^{1}$ L. De Paula, ${ }^{2}$ M. De Serio, ${ }^{14, j}$ P. De Simone, ${ }^{18}$ C. T. Dean, ${ }^{53}$ D. Decamp, ${ }^{4}$ L. Del Buono, ${ }^{8}$ B. Delaney, ${ }^{49}$ H.-P. Dembinski, ${ }^{11}$ M. Demmer, ${ }^{10}$ A. Dendek, ${ }^{30}$ D. Derkach,${ }^{37}$ O. Deschamps, ${ }^{5}$ F. Desse, ${ }^{7}$ F. Dettori, ${ }^{54}$ B. Dey ${ }^{65}$ A. Di Canto, ${ }^{42}$ P. Di Nezza, ${ }^{18}$ S. Didenko, ${ }^{70}$ H. Dijkstra, ${ }^{42}$ F. Dordei ${ }^{42}$ M. Dorigo, ${ }^{42, k}$ A. Dosil Suárez, ${ }^{41}$ L. Douglas, ${ }^{53}$ A. Dovbnya, ${ }^{45}$ K. Dreimanis,${ }^{54}$ L. Dufour ${ }^{27}$ G. Dujany, ${ }^{8}$ P. Durante, ${ }^{42}$ J. M. Durham, ${ }^{74}$ D. Dutta ${ }^{56}$ R. Dzhelyadin, ${ }^{39}$ M. Dziewiecki, ${ }^{12}$ A. Dziurda,${ }^{29}$ A. Dzyuba, ${ }^{33}$ S. Easo, ${ }^{51}$ U. Egede ${ }^{55}$ V. Egorychev, ${ }^{34}$ S. Eidelman, ${ }^{38, e}$ S. Eisenhardt, ${ }^{52}$ U. Eitschberger, ${ }^{10}$ R. Ekelhof, ${ }^{10}$ L. Eklund ${ }^{53}$ S. Ely, ${ }^{61}$ A. Ene, ${ }^{32}$ S. Escher, ${ }^{9}$ S. Esen,${ }^{27}$ T. Evans, ${ }^{59}$ A. Falabella, ${ }^{15}$ N. Farley,${ }^{47}$ S. Farry, ${ }^{54}$ D. Fazzini, ${ }^{20,42, \mathrm{c}}$ L. Federici ${ }^{25}$ P. Fernandez Declara, ${ }^{42}$ A. Fernandez Prieto, ${ }^{41}$ F. Ferrari, ${ }^{15}$ L. Ferreira Lopes, ${ }^{43}$ F. Ferreira Rodrigues, ${ }^{2}$ M. Ferro-Luzzi ${ }^{42}$ S. Filippov, ${ }^{36}$ R. A. Fini, ${ }^{14}$ M. Fiorini, ${ }^{16, a}$ M. Firlej, ${ }^{30}$ C. Fitzpatrick, ${ }^{43}$ T. Fiutowski, ${ }^{30}$ F. Fleuret,${ }^{7, b}$ M. Fontana, ${ }^{22,42}$ F. Fontanelli, ${ }^{19, \mathrm{~h}}$ R. Forty ${ }^{42}$ V. Franco Lima,${ }^{54}$ M. Frank, ${ }^{42}$ C. Frei,${ }^{42}$ J. Fu, ${ }^{21,1}$ W. Funk, ${ }^{42}$ C. Färber, ${ }^{42}$

M. Féo Pereira Rivello Carvalho, ${ }^{27}$ E. Gabriel, ${ }^{52}$ A. Gallas Torreira, ${ }^{41}$ D. Galli, ${ }^{15, \mathrm{~g}}$ S. Gallorini, ${ }^{23}$ S. Gambetta, ${ }^{52}$ Y. Gan, ${ }^{3}$ 
M. Gandelman, ${ }^{2}$ P. Gandini, ${ }^{21}$ Y. Gao, ${ }^{3}$ L. M. Garcia Martin, ${ }^{72}$ B. Garcia Plana, ${ }^{41}$ J. García Pardiñas, ${ }^{44}$ J. Garra Tico, ${ }^{49}$ L. Garrido ${ }^{40}$ D. Gascon, ${ }^{40}$ C. Gaspar, ${ }^{42}$ L. Gavardi,${ }^{10}$ G. Gazzoni, ${ }^{5}$ D. Gerick, ${ }^{12}$ E. Gersabeck, ${ }^{56}$ M. Gersabeck, ${ }^{56}$ T. Gershon, ${ }^{50}$ D. Gerstel, ${ }^{6}$ Ph. Ghez ${ }^{4}$ S. Gianì, ${ }^{43}$ V. Gibson, ${ }^{49}$ O. G. Girard, ${ }^{43}$ L. Giubega, ${ }^{32}$ K. Gizdov,${ }^{52}$ V. V. Gligorov, ${ }^{8}$ D. Golubkov, ${ }^{34}$ A. Golutvin, ${ }^{55,70}$ A. Gomes, ${ }^{1, \mathrm{~m}}$ I. V. Gorelov, ${ }^{35}$ C. Gotti, ${ }^{20, \mathrm{c}}$ E. Govorkova, ${ }^{27}$ J. P. Grabowski, ${ }^{12}$

R. Graciani Diaz ${ }^{40}$ L. A. Granado Cardoso, ${ }^{42}$ E. Graugés, ${ }^{40}$ E. Graverini,${ }^{44}$ G. Graziani, ${ }^{17}$ A. Grecu, ${ }^{32}$ R. Greim, ${ }^{27}$ P. Griffith, ${ }^{22}$ L. Grillo, ${ }^{56}$ L. Gruber, ${ }^{42}$ B. R. Gruberg Cazon,${ }^{57}$ O. Grünberg, ${ }^{67}$ C. Gu, ${ }^{3}$ E. Gushchin, ${ }^{36}$ Yu. Guz, ${ }^{39,42}$ T. Gys, ${ }^{42}$ C. Göbel, ${ }^{62}$ T. Hadavizadeh,${ }^{57}$ C. Hadjivasiliou, ${ }^{5}$ G. Haefeli, ${ }^{43}$ C. Haen, ${ }^{42}$ S. C. Haines, ${ }^{49}$ B. Hamilton,${ }^{60}$ X. Han, ${ }^{12}$ T. H. Hancock, ${ }^{57}$ S. Hansmann-Menzemer, ${ }^{12}$ N. Harnew, ${ }^{57}$ S. T. Harnew,${ }^{48}$ T. Harrison,${ }^{54}$ C. Hasse, ${ }^{42}$ M. Hatch,${ }^{42}$ J. He, ${ }^{63}$ M. Hecker ${ }^{55}$ K. Heinicke, ${ }^{10}$ A. Heister, ${ }^{10}$ K. Hennessy, ${ }^{54}$ L. Henry, ${ }^{72}$ E. van Herwijnen, ${ }^{42}$ M. Heß,${ }^{67}$ A. Hicheur, ${ }^{2}$ R. Hidalgo Charman, ${ }^{56}$ D. Hill, ${ }^{57}$ M. Hilton, ${ }^{56}$ P. H. Hopchev,${ }^{43}$ W. Hu, ${ }^{65}$ W. Huang, ${ }^{63}$ Z. C. Huard, ${ }^{59}$ W. Hulsbergen, ${ }^{27}$ T. Humair, ${ }^{55}$ M. Hushchyn, ${ }^{37}$ D. Hutchcroft, ${ }^{54}$ D. Hynds, ${ }^{27}$ P. Ibis, ${ }^{10}$ M. Idzik, ${ }^{30}$ P. Ilten, ${ }^{47}$ K. Ivshin, ${ }^{33}$ R. Jacobsson, ${ }^{42}$ J. Jalocha,${ }^{57}$ E. Jans, ${ }^{27}$ A. Jawahery, ${ }^{60}$ F. Jiang, ${ }^{3}$ M. John, ${ }^{57}$ D. Johnson, ${ }^{42}$ C. R. Jones, ${ }^{49}$ C. Joram, ${ }^{42}$ B. Jost, ${ }^{42}$ N. Jurik, ${ }^{57}$ S. Kandybei ${ }^{45}$ M. Karacson, ${ }^{42}$ J. M. Kariuki, ${ }^{48}$ S. Karodia,${ }^{53}$ N. Kazeev, ${ }^{37}$ M. Kecke, ${ }^{12}$ F. Keizer, ${ }^{49}$ M. Kelsey, ${ }^{61}$ M. Kenzie, ${ }^{49}$ T. Ketel, ${ }^{28}$ E. Khairullin, ${ }^{37}$ B. Khanji, ${ }^{12}$ C. Khurewathanakul, ${ }^{43}$ K. E. Kim, ${ }^{61}$ T. Kirn, ${ }^{9}$ S. Klaver, ${ }^{18}$ K. Klimaszewski, ${ }^{31}$ T. Klimkovich, ${ }^{11}$ S. Koliiev, ${ }^{46}$ M. Kolpin, ${ }^{12}$ R. Kopecna, ${ }^{12}$ P. Koppenburg, ${ }^{27}$ I. Kostiuk,${ }^{27}$ S. Kotriakhova, ${ }^{33}$ M. Kozeiha, ${ }^{5}$ L. Kravchuk, ${ }^{36}$ M. Kreps,${ }^{50}$ F. Kress, ${ }^{55}$ P. Krokovny, ${ }^{38, \mathrm{e}}$ W. Krupa,${ }^{30}$ W. Krzemien, ${ }^{31}$ W. Kucewicz ${ }^{29, n}$ M. Kucharczyk ${ }^{29}$ V. Kudryavtsev, ${ }^{38, e}$ A. K. Kuonen ${ }^{43}$ T. Kvaratskheliya, ${ }^{34,42}$ D. Lacarrere, ${ }^{42}$ G. Lafferty, ${ }^{56}$ A. Lai, ${ }^{22}$ D. Lancierini, ${ }^{44}$ G. Lanfranchi, ${ }^{18}$ C. Langenbruch, ${ }^{9}$ T. Latham, ${ }^{50}$ C. Lazzeroni, ${ }^{47}$ R. Le Gac,${ }^{6}$ A. Leflat, ${ }^{35}$ J. Lefrançois, ${ }^{7}$ R. Lefèvre, ${ }^{5}$ F. Lemaitre, ${ }^{42}$ O. Leroy, ${ }^{6}$ T. Lesiak, ${ }^{29}$ B. Leverington, ${ }^{12}$ P.-R. Li ${ }^{63}{ }^{6}$ T. Li,${ }^{3}$ Z. Li, ${ }^{61}$ X. Liang, ${ }^{61}$ T. Likhomanenko, ${ }^{69}$ R. Lindner, ${ }^{42}$ F. Lionetto, ${ }^{44}$ V. Lisovskyi, ${ }^{7}$ X. Liu, ${ }^{3}$ D. Loh, ${ }^{50}$ A. Loi,${ }^{22}$ I. Longstaff, ${ }^{53}$ J. H. Lopes, ${ }^{2}$ G. H. Lovell, ${ }^{49}$ D. Lucchesi, ${ }^{23, p}$ M. Lucio Martinez, ${ }^{41}$ A. Lupato, ${ }^{23}$ E. Luppi, ${ }^{16, a}$ O. Lupton, ${ }^{42}$ A. Lusiani, ${ }^{24}$ X. Lyu ${ }^{63}$ F. Machefert, ${ }^{7}$ F. Maciuc ${ }^{32}$ V. Macko, ${ }^{43}$ P. Mackowiak, ${ }^{10}$ S. Maddrell-Mander, ${ }^{48}$ O. Maev, ${ }^{33,42}$ K. Maguire, ${ }^{56}$ D. Maisuzenko, ${ }^{33}$ M. W. Majewski, ${ }^{30}$ S. Malde,${ }^{57}$ B. Malecki, ${ }^{29}$ A. Malinin, ${ }^{69}$ T. Maltsev, ${ }^{38, e}$ G. Manca, ${ }^{22, q}$ G. Mancinelli, ${ }^{6}$ D. Marangotto, ${ }^{21,1}$ J. Maratas, ${ }^{5, \mathrm{r}}$ J. F. Marchand, ${ }^{4}$ U. Marconi, ${ }^{15}$ C. Marin Benito, ${ }^{7}$ M. Marinangeli, ${ }^{43}$ P. Marino, ${ }^{43}$ J. Marks, ${ }^{12}$ P. J. Marshall,${ }^{54}$ G. Martellotti, ${ }^{26}$ M. Martin, ${ }^{6}$ M. Martinelli, ${ }^{42}$ D. Martinez Santos, ${ }^{41}$ F. Martinez Vidal, ${ }^{72}$ A. Massafferri, ${ }^{1}$ M. Materok, ${ }^{9}$ R. Matev, ${ }^{42}$ A. Mathad,${ }^{50}$ Z. Mathe,${ }^{42}$ C. Matteuzzi, ${ }^{20}$ A. Mauri, ${ }^{44}$ E. Maurice, ${ }^{7, b}$ B. Maurin,${ }^{43}$ A. Mazurov, ${ }^{47}$ M. McCann,${ }^{55,42}$ A. McNab,${ }^{56}$ R. McNulty,${ }^{13}$ J. V. Mead,${ }^{54}$ B. Meadows, ${ }^{59}$ C. Meaux, ${ }^{6}$ F. Meier,${ }^{10}$ N. Meinert, ${ }^{67}$ D. Melnychuk, ${ }^{31}$ M. Merk ${ }^{27}$ A. Merli, ${ }^{21,1}$ E. Michielin, ${ }^{23}$ D. A. Milanes ${ }^{66}$ E. Millard, ${ }^{50}$ M.-N. Minard, ${ }^{4}$ L. Minzoni, ${ }^{16, a}$ D. S. Mitzel, ${ }^{12}$ A. Mogini, ${ }^{8}$ J. Molina Rodriguez, ${ }^{1, \mathrm{~s}}$ T. Mombächer, ${ }^{10}$ I. A. Monroy, ${ }^{66}$ S. Monteil, ${ }^{5}$ M. Morandin, ${ }^{23}$ G. Morello, ${ }^{18}$ M. J. Morello, ${ }^{24, t}$ O. Morgunova, ${ }^{69}$ J. Moron, ${ }^{30}$ A. B. Morris, ${ }^{6}$ R. Mountain,${ }^{61}$ F. Muheim, ${ }^{52}$ M. Mulder, ${ }^{27}$ C. H. Murphy, ${ }^{57}$ D. Murray, ${ }^{56}$ A. Mödden, ${ }^{10}$ D. Müller, ${ }^{42}$ J. Müller, ${ }^{10}$ K. Müller, ${ }^{44}$ V. Müller, ${ }^{10}$ P. Naik, ${ }^{48}$ T. Nakada, ${ }^{43}$ R. Nandakumar, ${ }^{51}$ A. Nandi, ${ }^{57}$ T. Nanut, ${ }^{43}$ I. Nasteva, ${ }^{2}$ M. Needham, ${ }^{52}$ N. Neri, ${ }^{21}$ S. Neubert, ${ }^{12}$ N. Neufeld,${ }^{42}$ M. Neuner, ${ }^{12}$ T. D. Nguyen, ${ }^{43}$ C. Nguyen-Mau, ${ }^{43, u}$ S. Nieswand, ${ }^{9}$ R. Niet, ${ }^{10}$ N. Nikitin, ${ }^{35}$ A. Nogay, ${ }^{69}$ N. S. Nolte, ${ }^{42}$ D. P. O'Hanlon, ${ }^{15}$ A. Oblakowska-Mucha, ${ }^{30}$ V. Obraztsov, ${ }^{39}$ S. Ogilvy, ${ }^{18}$ R. Oldeman, ${ }^{22, q}$ C. J. G. Onderwater,${ }^{68}$ A. Ossowska, ${ }^{29}$ J. M. Otalora Goicochea, ${ }^{2}$ P. Owen, ${ }^{44}$ A. Oyanguren, ${ }^{72}$ P. R. Pais, ${ }^{43}$ T. Pajero, ${ }^{24, t}$ A. Palano, ${ }^{14}$ M. Palutan, ${ }^{18,42}$ G. Panshin, ${ }^{71}$ A. Papanestis, ${ }^{51}$ M. Pappagallo, ${ }^{52}$ L. L. Pappalardo, ${ }^{16, a}$ W. Parker,${ }^{60}$ C. Parkes, ${ }^{56}$ G. Passaleva, ${ }^{17,42}$ A. Pastore, ${ }^{14}$ M. Patel,${ }^{55}$ C. Patrignani, ${ }^{15,9}$ A. Pearce, ${ }^{42}$ A. Pellegrino, ${ }^{27}$ G. Penso, ${ }^{26}$ M. Pepe Altarelli, ${ }^{42}$ S. Perazzini,${ }^{42}$ D. Pereima ${ }^{34}$ P. Perret,${ }^{5}$ L. Pescatore,${ }^{43}$ K. Petridis, ${ }^{48}$ A. Petrolini, ${ }^{19}$ h A. Petrov,${ }^{69}$ S. Petrucci, ${ }^{52}$ M. Petruzzo, ${ }^{21,1}$ B. Pietrzyk, ${ }^{4}$ G. Pietrzyk, ${ }^{43}$ M. Pikies, ${ }^{29}$ M. Pili,${ }^{57}$ D. Pinci, ${ }^{26}$ J. Pinzino, ${ }^{42}$ F. Pisani, ${ }^{42, o}$ A. Piucci, ${ }^{12}$ V. Placinta, ${ }^{32}$ S. Playfer,${ }^{52}$ J. Plews, ${ }^{47}$ M. Plo Casasus, ${ }^{41}$ F. Polci, ${ }^{8}$ M. Poli Lener, ${ }^{18}$ A. Poluektov, ${ }^{50}$ N. Polukhina,${ }^{70, v}$ I. Polyakov, ${ }^{61}$ E. Polycarpo, ${ }^{2}$ G. J. Pomery,${ }^{48}$ S. Ponce, ${ }^{42}$ A. Popov,${ }^{39}$ D. Popov,${ }^{47,11}$ S. Poslavskii, ${ }^{39}$ C. Potterat, ${ }^{2}$ E. Price, ${ }^{48}$ J. Prisciandaro, ${ }^{41}$ C. Prouve, ${ }^{48}$ V. Pugatch,${ }^{46}$ A. Puig Navarro, ${ }^{44}$ H. Pullen, ${ }^{57}$ G. Punzi, ${ }^{24 i}$ W. Qian, ${ }^{63}$ J. Qin, ${ }^{63}$ R. Quagliani, ${ }^{8}$ B. Quintana, ${ }^{5}$ B. Rachwal, ${ }^{30}$ J. H. Rademacker, ${ }^{48}$ M. Rama, ${ }^{24}$ M. Ramos Pernas, ${ }^{41}$ M. S. Rangel, ${ }^{2}$ F. Ratnikov, ${ }^{37, w}$ G. Raven ${ }^{28}$ M. Ravonel Salzgeber, ${ }^{42}$ M. Reboud, ${ }^{4}$ F. Redi, ${ }^{43}$ S. Reichert, ${ }^{10}$ A. C. dos Reis, ${ }^{1}$ F. Reiss, ${ }^{8}$ C. Remon Alepuz,${ }^{72}$ Z. Ren, ${ }^{3}$ V. Renaudin, ${ }^{7}$ S. Ricciardi,${ }^{51}$ S. Richards,${ }^{48}$ K. Rinnert,${ }^{54}$ P. Robbe, ${ }^{7}$ A. Robert, ${ }^{8}$ A. B. Rodrigues,${ }^{43}$ E. Rodrigues,${ }^{59}$

J. A. Rodriguez Lopez, ${ }^{66}$ M. Roehrken, ${ }^{42}$ A. Rogozhnikov, ${ }^{37}$ S. Roiser, ${ }^{42}$ A. Rollings, ${ }^{57}$ V. Romanovskiy, ${ }^{39}$

A. Romero Vidal, ${ }^{41}$ M. Rotondo, ${ }^{18}$ M. S. Rudolph ${ }^{61}$ T. Ruf, ${ }^{42}$ J. Ruiz Vidal, ${ }^{72}$ J. J. Saborido Silva, ${ }^{41}$ N. Sagidova, ${ }^{33}$

B. Saitta, ${ }^{22, q}$ V. Salustino Guimaraes, ${ }^{62}$ C. Sanchez Gras, ${ }^{27}$ C. Sanchez Mayordomo, ${ }^{72}$ B. Sanmartin Sedes,${ }^{41}$

R. Santacesaria, ${ }^{26}$ C. Santamarina Rios, ${ }^{41}$ M. Santimaria, ${ }^{18}$ E. Santovetti, ${ }^{25, x}$ G. Sarpis, ${ }^{56}$ A. Sarti, ${ }^{18, y}$ C. Satriano, ${ }^{26, z}$ 
A. Satta, ${ }^{25}$ M. Saur, ${ }^{63}$ D. Savrina, ${ }^{34,35}$ S. Schael,${ }^{9}$ M. Schellenberg, ${ }^{10}$ M. Schiller, ${ }^{53}$ H. Schindler, ${ }^{42}$ M. Schmelling, ${ }^{11}$ T. Schmelzer, ${ }^{10}$ B. Schmidt, ${ }^{42}$ O. Schneider, ${ }^{43}$ A. Schopper, ${ }^{42}$ H. F. Schreiner, ${ }^{59}$ M. Schubiger, ${ }^{43}$ M. H. Schune, ${ }^{7}$ R. Schwemmer, ${ }^{42}$ B. Sciascia, ${ }^{18}$ A. Sciubba, ${ }^{26, y}$ A. Semennikov, ${ }^{34}$ E. S. Sepulveda, ${ }^{8}$ A. Sergi, ${ }^{47,42}$ N. Serra, ${ }^{44}$ J. Serrano, ${ }^{6}$ L. Sestini, ${ }^{23}$ A. Seuthe, ${ }^{10}$ P. Seyfert, ${ }^{42}$ M. Shapkin, ${ }^{39}$ Y. Shcheglov, ${ }^{33}$ T. Shears, ${ }^{54}$ L. Shekhtman, ${ }^{38, e}$ V. Shevchenko, ${ }^{69}$ E. Shmanin, ${ }^{70}$ B. G. Siddi, ${ }^{16}$ R. Silva Coutinho, ${ }^{44}$ L. Silva de Oliveira, ${ }^{2}$ G. Simi ${ }^{23, p}$ S. Simone, ${ }^{14, j}$ N. Skidmore, ${ }^{12}$ T. Skwarnicki, ${ }^{61}$ J. G. Smeaton, ${ }^{49}$ E. Smith, ${ }^{9}$ I. T. Smith, ${ }^{52}$ M. Smith,${ }^{55}$ M. Soares, ${ }^{15}$ 1. Soares Lavra, ${ }^{1}$ M. D. Sokoloff, ${ }^{59}$ F. J. P. Soler, ${ }^{53}$ B. Souza De Paula, ${ }^{2}$ B. Spaan, ${ }^{10}$ P. Spradlin,${ }^{53}$ F. Stagni,${ }^{42}$ M. Stahl,${ }^{12}$ S. Stahl,${ }^{42}$ P. Stefko, ${ }^{43}$ S. Stefkova,${ }^{55}$ O. Steinkamp, ${ }^{44}$ S. Stemmle, ${ }^{12}$ O. Stenyakin, ${ }^{39}$ M. Stepanova, ${ }^{33}$ H. Stevens, ${ }^{10}$ A. Stocchi, ${ }^{7}$ S. Stone ${ }^{61}$ B. Storaci, ${ }^{44}$ S. Stracka, ${ }^{24, i}$ M. E. Stramaglia, ${ }^{43}$ M. Straticiuc, ${ }^{32}$ U. Straumann, ${ }^{44}$ S. Strokov, ${ }^{71}$ J. Sun, ${ }^{3}$ L. Sun, ${ }^{64}$ K. Swientek, ${ }^{30}$ V. Syropoulos, ${ }^{28}$ T. Szumlak, ${ }^{30}$ M. Szymanski, ${ }^{63}$ S. T'Jampens, ${ }^{4}$ Z. Tang, ${ }^{3}$ A. Tayduganov, ${ }^{6}$ T. Tekampe, ${ }^{10}$ G. Tellarini, ${ }^{16}$ F. Teubert, ${ }^{42}$ E. Thomas, ${ }^{42}$ J. van Tilburg, ${ }^{27}$ M. J. Tilley, ${ }^{55}$ V. Tisserand, ${ }^{5}$ M. Tobin, ${ }^{30}$ S. Tolk, ${ }^{42}$ L. Tomassetti, ${ }^{16, a}$ D. Tonelli, ${ }^{24}$ D. Y. Tou,${ }^{8}$ R. Tourinho Jadallah Aoude, ${ }^{1}$ E. Tournefier, ${ }^{4}$ M. Traill,${ }^{53}$ M. T. Tran, ${ }^{43}$ A. Trisovic, ${ }^{49}$ A. Tsaregorodtsev, ${ }^{6}$ G. Tuci, ${ }^{24}$ A. Tully, ${ }^{49}$ N. Tuning, ${ }^{27,42}$ A. Ukleja, ${ }^{31}$ A. Usachov, ${ }^{7}$ A. Ustyuzhanin, ${ }^{37}$ U. Uwer, ${ }^{12}$ A. Vagner ${ }^{71}$ V. Vagnoni, ${ }^{15}$ A. Valassi ${ }^{42}$ S. Valat ${ }^{42}$ G. Valenti, ${ }^{15}$ R. Vazquez Gomez, ${ }^{42}$ P. Vazquez Regueiro, ${ }^{41}$ S. Vecchi, ${ }^{16}$ M. van Veghel, ${ }^{27}$ J. J. Velthuis, ${ }^{48}$ M. Veltri, ${ }^{17, a a}$ G. Veneziano, ${ }^{57}$ A. Venkateswaran, ${ }^{61}$ T. A. Verlage, ${ }^{9}$ M. Vernet,${ }^{5}$ M. Veronesi, ${ }^{27}$ N. V. Veronika, ${ }^{13}$ M. Vesterinen,${ }^{57}$ J. V. Viana Barbosa, ${ }^{42}$ D. Vieira, ${ }^{63}$ M. Vieites Diaz,${ }^{41}$ H. Viemann, ${ }^{67}$ X. Vilasis-Cardona, ${ }^{40, f}$ A. Vitkovskiy, ${ }^{27}$ M. Vitti, ${ }^{49}$ V. Volkov, ${ }^{35}$ A. Vollhardt, ${ }^{44}$ B. Voneki, ${ }^{42}$ A. Vorobyev, ${ }^{33}$ V. Vorobyev,,${ }^{38, e}$ J. A. de Vries, ${ }^{27}$ C. Vázquez Sierra, ${ }^{27}$ R. Waldi, ${ }^{67}$ J. Walsh, ${ }^{24}$ J. Wang, ${ }^{61}$ M. Wang, ${ }^{3}$ Y. Wang, ${ }^{65}$ Z. Wang, ${ }^{44}$ D. R. Ward, ${ }^{49}$ H. M. Wark, ${ }^{54}$ N. K. Watson, ${ }^{47}$ D. Websdale, ${ }^{55}$ A. Weiden, ${ }^{44}$ C. Weisser, ${ }^{58}$ M. Whitehead, ${ }^{9}$ J. Wicht,${ }^{50}$ G. Wilkinson, ${ }^{57}$ M. Wilkinson, ${ }^{61}$ I. Williams, ${ }^{49}$ M. R. J. Williams, ${ }^{56}$ M. Williams, ${ }^{58}$ T. Williams, ${ }^{47}$ F. F. Wilson, ${ }^{51,42}$ J. Wimberley, ${ }^{60}$ M. Winn, ${ }^{7}$ J. Wishahi, ${ }^{10}$ W. Wislicki, ${ }^{31}$ M. Witek, ${ }^{29}$ G. Wormser, ${ }^{7}$ S. A. Wotton, ${ }^{49}$ K. Wyllie, ${ }^{42}$ D. Xiao, ${ }^{65}$ Y. Xie,${ }^{65}$ A. Xu, ${ }^{3}$ M. Xu, ${ }^{65}$ Q. Xu, ${ }^{63}$ Z. Xu, ${ }^{3}$ Z. Xu, ${ }^{4}$ Z. Yang, ${ }^{3}$ Z. Yang, ${ }^{60}$ Y. Yao, ${ }^{61}$ L. E. Yeomans,${ }^{54}$ H. Yin,${ }^{65}$ J. Yu, ${ }^{65, b b}$ X. Yuan, ${ }^{61}$ O. Yushchenko, ${ }^{39}$ K. A. Zarebski, ${ }^{47}$ M. Zavertyaev, ${ }^{11, v}$ D. Zhang, ${ }^{65}$ L. Zhang, ${ }^{3}$ W. C. Zhang, ${ }^{3, c c}$ Y. Zhang, ${ }^{7}$ A. Zhelezov, ${ }^{12}$ Y. Zheng, ${ }^{63}$ X. Zhu, ${ }^{3}$ V. Zhukov, ${ }^{9,35}$ J. B. Zonneveld, ${ }^{52}$ and S. Zucchelli ${ }^{15}$

\title{
(LHCb Collaboration)
}

\author{
${ }^{1}$ Centro Brasileiro de Pesquisas Físicas $(C B P F)$, Rio de Janeiro, Brazil \\ ${ }^{2}$ Universidade Federal do Rio de Janeiro (UFRJ), Rio de Janeiro, Brazil \\ ${ }^{3}$ Center for High Energy Physics, Tsinghua University, Beijing, China \\ ${ }^{4}$ Univ. Grenoble Alpes, Univ. Savoie Mont Blanc, CNRS, IN2P3-LAPP, Annecy, France \\ ${ }^{5}$ Clermont Université, Université Blaise Pascal, CNRS/IN2P3, LPC, Clermont-Ferrand, France \\ ${ }^{6}$ Aix Marseille Univ, CNRS/IN2P3, CPPM, Marseille, France \\ ${ }^{7}$ LAL, Univ. Paris-Sud, CNRS/IN2P3, Université Paris-Saclay, Orsay, France \\ ${ }^{8}$ LPNHE, Sorbonne Université, Paris Diderot Sorbonne Paris Cité, CNRS/IN2P3, Paris, France \\ ${ }^{9}$ I. Physikalisches Institut, RWTH Aachen University, Aachen, Germany \\ ${ }^{10}$ Fakultät Physik, Technische Universität Dortmund, Dortmund, Germany \\ ${ }^{11}$ Max-Planck-Institut für Kernphysik (MPIK), Heidelberg, Germany \\ ${ }^{12}$ Physikalisches Institut, Ruprecht-Karls-Universität Heidelberg, Heidelberg, Germany \\ ${ }^{13}$ School of Physics, University College Dublin, Dublin, Ireland \\ ${ }^{14}$ INFN Sezione di Bari, Bari, Italy \\ ${ }^{15}$ INFN Sezione di Bologna, Bologna, Italy \\ ${ }^{16}$ INFN Sezione di Ferrara, Ferrara, Italy \\ ${ }^{17}$ INFN Sezione di Firenze, Firenze, Italy \\ ${ }^{18}$ INFN Laboratori Nazionali di Frascati, Frascati, Italy \\ ${ }^{19}$ INFN Sezione di Genova, Genova, Italy \\ ${ }^{20}$ INFN Sezione di Milano-Bicocca, Milano, Italy \\ ${ }^{21}$ INFN Sezione di Milano, Milano, Italy \\ ${ }^{22}$ INFN Sezione di Cagliari, Monserrato, Italy \\ ${ }^{23}$ INFN Sezione di Padova, Padova, Italy \\ ${ }^{24}$ INFN Sezione di Pisa, Pisa, Italy \\ ${ }^{25}$ INFN Sezione di Roma Tor Vergata, Roma, Italy \\ ${ }^{26}$ INFN Sezione di Roma La Sapienza, Roma, Italy
}




\footnotetext{
${ }^{27}$ Nikhef National Institute for Subatomic Physics, Amsterdam, Netherlands

${ }^{28}$ Nikhef National Institute for Subatomic Physics and VU University Amsterdam, Amsterdam, Netherlands

${ }^{29}$ Henryk Niewodniczanski Institute of Nuclear Physics Polish Academy of Sciences, Kraków, Poland

${ }^{30}$ AGH-University of Science and Technology, Faculty of Physics and Applied Computer Science, Kraków, Poland

${ }^{31}$ National Center for Nuclear Research (NCBJ), Warsaw, Poland

${ }^{32}$ Horia Hulubei National Institute of Physics and Nuclear Engineering, Bucharest-Magurele, Romania

${ }^{33}$ Petersburg Nuclear Physics Institute (PNPI), Gatchina, Russia

${ }^{34}$ Institute of Theoretical and Experimental Physics (ITEP), Moscow, Russia

${ }^{35}$ Institute of Nuclear Physics, Moscow State University (SINP MSU), Moscow, Russia

${ }^{36}$ Institute for Nuclear Research of the Russian Academy of Sciences (INR RAS), Moscow, Russia

${ }^{37}$ Yandex School of Data Analysis, Moscow, Russia

${ }^{38}$ Budker Institute of Nuclear Physics (SB RAS), Novosibirsk, Russia

${ }^{39}$ Institute for High Energy Physics (IHEP), Protvino, Russia

${ }^{40}$ ICCUB, Universitat de Barcelona, Barcelona, Spain

${ }^{41}$ Instituto Galego de Física de Altas Enerxías (IGFAE), Universidade de Santiago de Compostela, Santiago de Compostela, Spain

${ }^{42}$ European Organization for Nuclear Research (CERN), Geneva, Switzerland

${ }^{43}$ Institute of Physics, Ecole Polytechnique Fédérale de Lausanne (EPFL), Lausanne, Switzerland

${ }^{44}$ Physik-Institut, Universität Zürich, Zürich, Switzerland

${ }^{45}$ NSC Kharkiv Institute of Physics and Technology (NSC KIPT), Kharkiv, Ukraine

${ }^{46}$ Institute for Nuclear Research of the National Academy of Sciences (KINR), Kyiv, Ukraine

${ }^{47}$ University of Birmingham, Birmingham, United Kingdom

${ }^{48}$ H.H. Wills Physics Laboratory, University of Bristol, Bristol, United Kingdom

${ }^{49}$ Cavendish Laboratory, University of Cambridge, Cambridge, United Kingdom

${ }^{50}$ Department of Physics, University of Warwick, Coventry, United Kingdom

${ }^{51}$ STFC Rutherford Appleton Laboratory, Didcot, United Kingdom

${ }^{52}$ School of Physics and Astronomy, University of Edinburgh, Edinburgh, United Kingdom

${ }^{53}$ School of Physics and Astronomy, University of Glasgow, Glasgow, United Kingdom

${ }^{54}$ Oliver Lodge Laboratory, University of Liverpool, Liverpool, United Kingdom

${ }^{55}$ Imperial College London, London, United Kingdom

${ }^{56}$ School of Physics and Astronomy, University of Manchester, Manchester, United Kingdom

${ }^{57}$ Department of Physics, University of Oxford, Oxford, United Kingdom

${ }^{58}$ Massachusetts Institute of Technology, Cambridge, Massachusetts, USA

${ }^{59}$ University of Cincinnati, Cincinnati, Ohio, USA

${ }^{60}$ University of Maryland, College Park, Maryland, USA

${ }^{61}$ Syracuse University, Syracuse, New York, USA

${ }^{62}$ Pontifícia Universidade Católica do Rio de Janeiro (PUC-Rio), Rio de Janeiro, Brazil

(associated with Universidade Federal do Rio de Janeiro (UFRJ), Rio de Janeiro, Brazil)

${ }^{63}$ University of Chinese Academy of Sciences, Beijing, China

(associated with Center for High Energy Physics, Tsinghua University, Beijing, China)

${ }^{64}$ School of Physics and Technology, Wuhan University, Wuhan, China

(associated with Center for High Energy Physics, Tsinghua University, Beijing, China)

${ }^{65}$ Institute of Particle Physics, Central China Normal University, Wuhan, Hubei, China

(associated with Center for High Energy Physics, Tsinghua University, Beijing, China)

${ }^{66}$ Departamento de Fisica, Universidad Nacional de Colombia, Bogota, Colombia

(associated with LPNHE, Sorbonne Université, Paris Diderot Sorbonne Paris Cité, CNRS/IN2P3, Paris, France)

${ }^{67}$ Institut für Physik, Universität Rostock, Rostock, Germany

(associated with Physikalisches Institut, Ruprecht-Karls-Universität Heidelberg, Heidelberg, Germany)

${ }^{68}$ Van Swinderen Institute, University of Groningen, Groningen, Netherlands

(associated with Nikhef National Institute for Subatomic Physics, Amsterdam, Netherlands)

${ }^{69}$ National Research Centre Kurchatov Institute, Moscow, Russia

(associated with Institute of Theoretical and Experimental Physics (ITEP), Moscow, Russia)

${ }^{70}$ National University of Science and Technology "MISIS", Moscow, Russia

(associated with Institute of Theoretical and Experimental Physics (ITEP), Moscow, Russia)

${ }^{71}$ National Research Tomsk Polytechnic University, Tomsk, Russia

(associated with Institute of Theoretical and Experimental Physics (ITEP), Moscow, Russia)

${ }^{72}$ Instituto de Fisica Corpuscular, Centro Mixto Universidad de Valencia-CSIC, Valencia, Spain

(associated with ICCUB, Universitat de Barcelona, Barcelona, Spain)

${ }^{73}$ University of Michigan, Ann Arbor, USA

(associated with Syracuse University, Syracuse, New York, USA)
} 


\section{${ }^{74}$ Los Alamos National Laboratory (LANL), Los Alamos, USA \\ (associated with Syracuse University, Syracuse, New York, USA)}

${ }^{a}$ Also at Università di Ferrara, Ferrara, Italy.

${ }^{\mathrm{b}}$ Also at Laboratoire Leprince-Ringuet, Palaiseau, France.

${ }^{c}$ Also at Università di Milano Bicocca, Milano, Italy.

${ }^{\mathrm{d}}$ Also at Università di Modena e Reggio Emilia, Modena, Italy.

${ }^{\mathrm{e}}$ Also at Novosibirsk State University, Novosibirsk, Russia.

${ }^{\mathrm{f}}$ Also at LIFAELS, La Salle, Universitat Ramon Llull, Barcelona, Spain.

${ }^{\mathrm{g}}$ Also at Università di Bologna, Bologna, Italy.

${ }^{\mathrm{h}}$ Also at Università di Genova, Genova, Italy.

${ }^{i}$ Also at Università di Pisa, Pisa, Italy.

${ }^{\mathrm{j}}$ Also at Università di Bari, Bari, Italy.

${ }^{\mathrm{k}}$ Also at Sezione INFN di Trieste, Trieste, Italy.

${ }^{1}$ Also at Università degli Studi di Milano, Milano, Italy.

${ }^{\mathrm{m}}$ Also at Universidade Federal do Triângulo Mineiro (UFTM), Uberaba-MG, Brazil.

${ }^{\mathrm{n}}$ Also at AGH-University of Science and Technology, Faculty of Computer Science, Electronics and Telecommunications, Kraków, Poland.

${ }^{\circ}$ Also at INFN Sezione di Bologna, Bologna, Italy

${ }^{\mathrm{p}}$ Also at Università di Padova, Padova, Italy.

${ }^{\mathrm{q}}$ Also at Università di Cagliari, Cagliari, Italy.

${ }^{r}$ Also at MSU-Iligan Institute of Technology (MSU-IIT), Iligan, Philippines.

${ }^{\mathrm{s}}$ Also at Escuela Agrícola Panamericana, San Antonio de Oriente, Honduras.

${ }^{\mathrm{t}}$ Also at Scuola Normale Superiore, Pisa, Italy.

"Also at Hanoi University of Science, Hanoi, Vietnam.

${ }^{v}$ Also at P.N. Lebedev Physical Institute, Russian Academy of Science (LPI RAS), Moscow, Russia.

${ }^{w}$ Also at National Research University Higher School of Economics, Moscow, Russia.

${ }^{x}$ Also at Università di Roma Tor Vergata, Roma, Italy.

${ }^{\mathrm{y}}$ Also at Università di Roma La Sapienza, Roma, Italy.

${ }^{\mathrm{z}}$ Also at Università della Basilicata, Potenza, Italy.

${ }^{\text {aa } A l s o ~ a t ~ U n i v e r s i t a ̀ ~ d i ~ U r b i n o, ~ U r b i n o, ~ I t a l y . ~}$

${ }^{\mathrm{bb}}$ Also at Physics and Micro Electronic College, Hunan University, Changsha City, China.

${ }^{\mathrm{cc}}$ Also at School of Physics and Information Technology, Shaanxi Normal University (SNNU), Xi'an, China. 